\title{
A modified lightwand-guided approach for placement of a King Laryngeal Tube ${ }^{\mathrm{TM}}$
}

\author{
Dannielle Tinker, HBA (D) - Rosie Earle, BSc, MD · Himat Vaghadia, MBBS, FRCPC
}

Received: 14 September 2017/ Accepted: 31 October 2017/Published online: 8 November 2017

(C) Canadian Anesthesiologists' Society 2017

\section{To the Editor,}

The King Laryngeal Tube ${ }^{\mathrm{TM}}$ (King LT-D; King Systems, Noblesville, IN, USA) is a single-lumen supraglottic airway (SGA) device with multiple ventilation outlets, flanked by a proximal oropharyngeal and distal esophageal cuffs. ${ }^{1-3}$ Compared with other SGAs, the King LT-D offers a comparable proximal airway seal with the benefit of a distal cuff in the esophagus acting as a barrier to aspiration of gastric contents. ${ }^{2}$ The King LT-D can be used for emergency and elective airway management. ${ }^{3}$

Insertion of the King LT-D, as described by the manufacturer, requires the clinician to advance it blindly into the oropharynx until the base of the connector is aligned with the teeth, inflate the cuffs, attach the circuit, and bag the patient whilst withdrawing the device until ventilation is free flowing. Many experienced anesthesiologists and trainees find this method to be technically difficult and correct positioning of the device for ventilation is not always assured.

Mahajan et al. suggested a lightwand-based solution for a similar SGA, the double-lumen VBM Medizintechnik LTS II ${ }^{\circledR}$ airway (VBM Medizintechnik GmbH, Sulz am Neckar, Germany). A lighted stylet is inserted into the suction channel of the LTS-II and correct placement of the LTS II is confirmed by observing a diffuse dim glow in the anterior neck at the level of the tracheal cartilage. ${ }^{4}$ Some concerns with this approach include: the risk of trauma to the airway if the lighted stylet accidentally protrudes from

D. Tinker, HBA $(\varangle) \cdot$ R. Earle, BSc, MD .

H. Vaghadia, MBBS, FRCPC

Department of Anaesthesia, Vancouver Coastal Health,

Vancouver, BC, Canada

e-mail: dannielleleahtinker@gmail.com the distal end of the LTS II during advancement; the diffuse dim glow can be obscured because light has to traverse the walls of the esophagus, trachea, and soft tissues of the neck; and, correct LTS II position cannot be assured because the anterior neck glow is an imprecise end point for correct supraglottic placement of the LTS II's ventilation outlets. Unfortunately, this method is unsuitable for standard King LT-Ds that do not have a suction channel.

We suggest a modification to the above approach, which was evaluated with the King LT-D. This method involves inserting the flexible lightwand (lighted intubation stylet 16", Bovie Medical Corporation, Clearwater, FL, USA) into the ventilation lumen of the King LT-D until its tip is at the ventilation outlet (Figure, panels A and B). The King LT-D is then advanced into the oropharynx until transillumination of a bright central focus is visualized at the level of the thyroid cartilage, with a uni- or bilateral smaller foci seen on either side caused by light passing through the lateral ventilation eyes (Figure, panel C), confirming precise supraglottic placement of the ventilation outlets.

Our modified method can easily be practiced as a solo clinician, eliminates tissue trauma risk from lightwand advancement through the suction channel, and reduces adjustment and manipulation of the device to confirm placement. Using this modified lightwand method we have performed more than 30 successful King LT-D placements over the last three months. We observed a clear midline and lateral glow in all cases and repositioning of the King LT-D was not required in any case. The LTS II airway was unavailable at our institution; we do, however, believe that the method would work equally as well on both King LT-D and LTS II SGAs. We encourage anesthesiologists who 


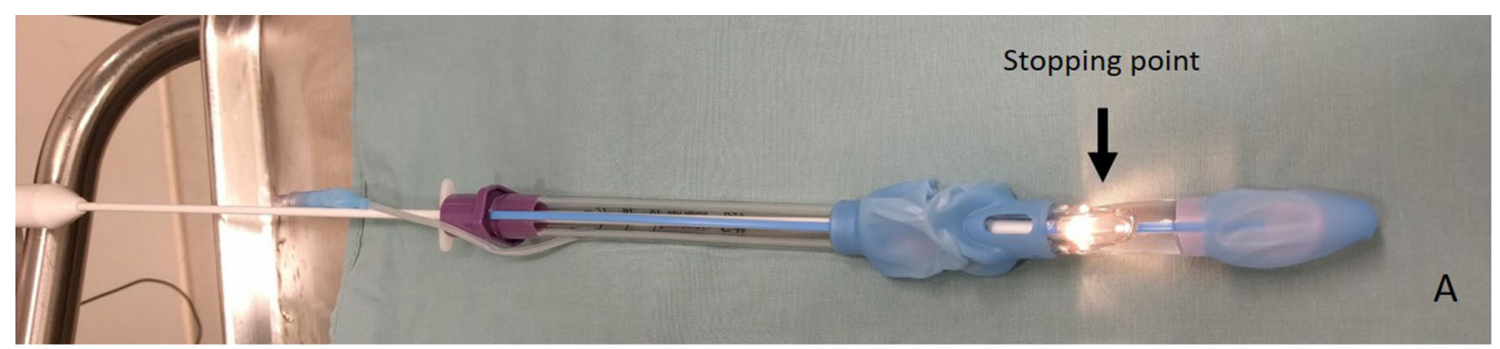

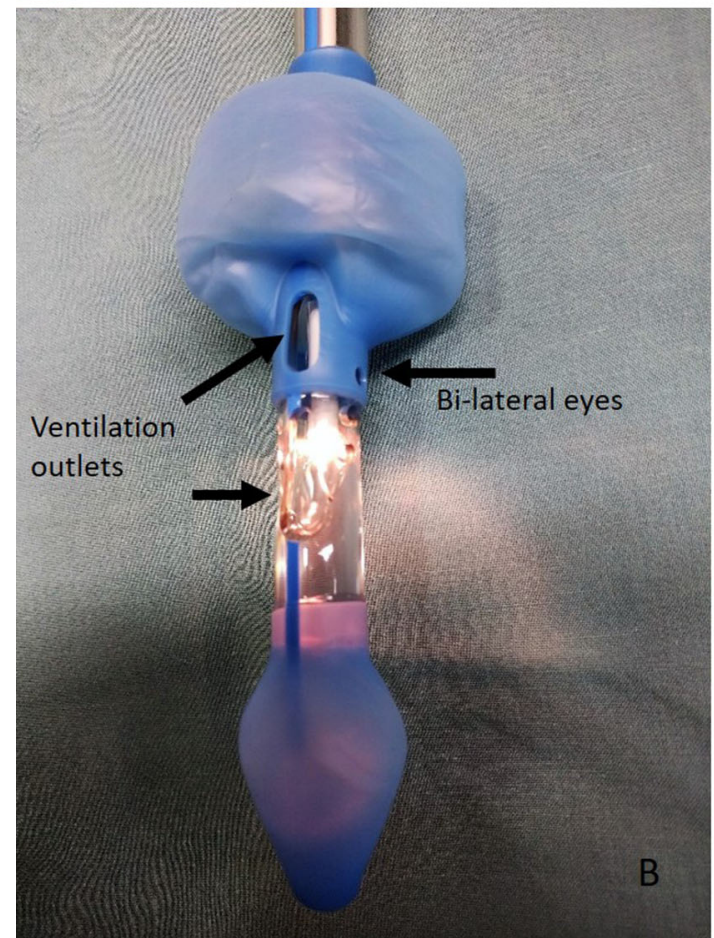

Figure The King Laryngeal Tube ${ }^{\mathrm{TM}}$ (King LT-D; King Systems, Noblesville, IN, USA) pre-insertion and during insertion. Panel A) King LT-D with lightwand inserted to stopping point at the main ventilation outlet. Panel B) Main ventilation outlets and additional symmetric bi-lateral eye outlets (left eye outlet visible in photo).

experience difficulty with King LT-D placement to consider our modified lightwand method.

Competing interests This work was supported by Internal Departmental Funds. The other authors have no conflicts.

\section{References}

1. Brimacombe J, Keller C, Brimacombe L. A comparison of the laryngeal mask airway ProSeal and the laryngeal tube airway in

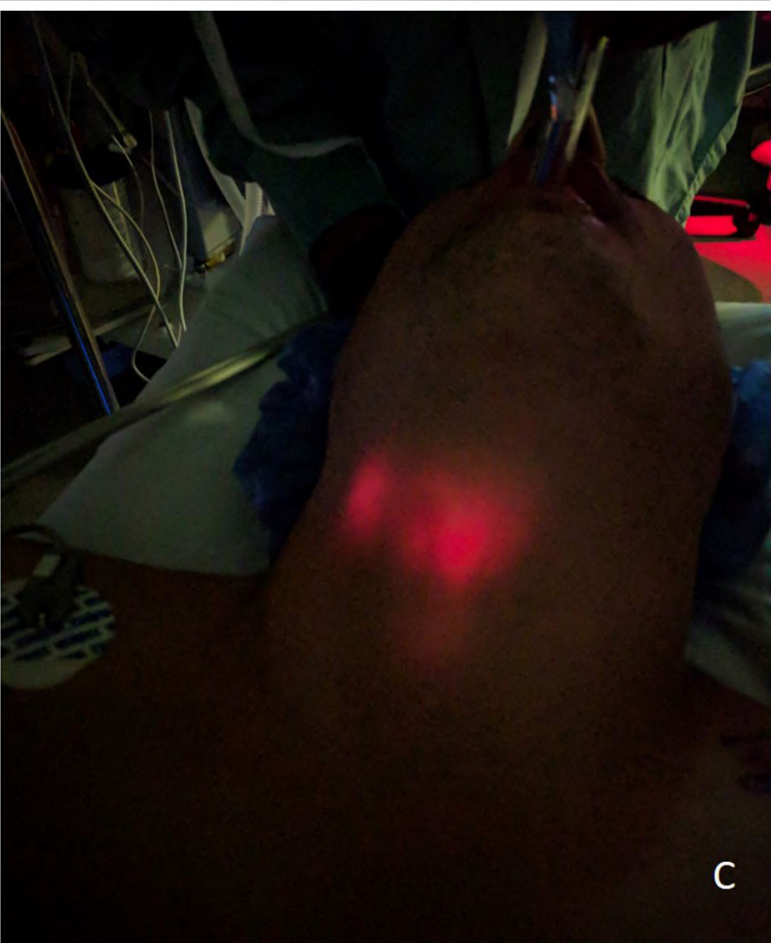

Panel C) The King LT-D's position is confirmed with transillumination in a patient at the level of the thyroid cartilage with one midline foci from the ventilation outlet and one lateral foci from one of the bilateral eye outlets

paralyzed anesthetized adult patients undergoing pressurecontrolled ventilation. Anesth Analg 2002; 95: 770-6.

2. Ramaiah $R$, Das D, Bhananker SM, Joffe AM. Extraglottic airway devices: a review. Int J Crit Illn Inj Sci 2014; 4: 77-87.

3. Dörges V, Ocker $H$, Wenzel V, Steinfath $M$, Gerlach $K$. The Laryngeal Tube $S$ : a new simple airway device. Anesth Analg 2003; 96: 618-21.

4. Mahajan R, Nazir R, Jatinder M, Gulati S. Lightwand guided insertion of laryngeal tube suction. Anaesthesia 2015; 70: 1219-20.

5. Asai T, Murao K, Shingu $K$. Efficacy of the laryngeal tube during intermittent positive-pressure ventilation. Anaesthesia 2000; 55: 1099-102. 